\title{
Countercurrent Air-Water Flow in a Scale-Down Model of a Pressurizer Surge Line
}

\author{
Takashi Futatsugi, ${ }^{1}$ Chihiro Yanagi, ${ }^{2}$ Michio Murase, ${ }^{2}$ \\ Shigeo Hosokawa, ${ }^{1}$ and Akio Tomiyama ${ }^{1}$ \\ ${ }^{1}$ Department of Mechanical Engineering, Faculty of Engineering, Kobe University, 1-1 Rokkodai, Nada, Hyogo Kobe,
657-8501, Japan
${ }^{2}$ Institute of Nuclear Safety System, Inc. (INSS), 64 Sata, Mihama-cho, Mikata-gun, Fukui 919-1205, Japan
}

Correspondence should be addressed to Akio Tomiyama, tomiyama@mech.kobe-u.ac.jp

Received 13 October 2011; Accepted 16 December 2011

Academic Editor: Thomas Hoehne

Copyright ( $) 2012$ Takashi Futatsugi et al. This is an open access article distributed under the Creative Commons Attribution License, which permits unrestricted use, distribution, and reproduction in any medium, provided the original work is properly cited.

\begin{abstract}
Steam generated in a reactor core and water condensed in a pressurizer form a countercurrent flow in a surge line between a hot leg and the pressurizer during reflux cooling. Characteristics of countercurrent flow limitation (CCFL) in a 1/10-scale model of the surge line were measured using air and water at atmospheric pressure and room temperature. The experimental results show that CCFL takes place at three different locations, that is, at the upper junction, in the surge line, and at the lower junction, and its characteristics are governed by the most dominating flow limitation among the three. Effects of inclination angle and elbows of the surge line on CCFL characteristics were also investigated experimentally. The effects of inclination angle on CCFL depend on the flow direction, that is, the effect is large for the nearly horizontal flow and small for the vertical flow at the upper junction. The presence of elbows increases the flow limitation in the surge line, whereas the flow limitations at the upper and lower junctions do not depend on the presence of elbows.
\end{abstract}

\section{Introduction}

The mid-loop operation is to be conducted during plant refueling and maintenance of a PWR (Pressurized Water Reactor). In this operation, the reactor coolant level is kept around the primary loop center, and decay heat is removed by RHR (Residual Heat Removal) systems. If the loss of cooling systems such as RHR and/or other cooling systems takes place, cooling water in the reactor core may be heated up to boil and the top of the fuel assembly can be exposed to the air. In such an event, reflux cooling by the steam generators (SG) is regarded as one of the possible and effective core cooling methods. The reflux cooling is a way of core cooling by making use of water condensed in SGs. The steam generated in the reactor core and water condensed in the SG form a countercurrent flow in the hot leg. The authors therefore measured CCFL (Countercurrent Flow Limitation) characteristics in a scale-down model of a hot leg using air and water [1] and reported that CCFL can be accurately evaluated based on a one dimensional momentum balance for air-water two-phase flow [2]. In addition to this CCFL, the steam generated in the reactor core and water condensed in the pressurizer due to heat transfer to the vessel wall may also form a countercurrent flow in a surge line which connects the hot leg and the pressurizer. The ROSA-IV/LSTF (Rig-of-Safety-Assessment No. 4/Large Scale Test Facility) experiment [3], which simulated the loss of RHR systems during mid-loop operation, reported that water actually accumulated in the pressurizer due to CCFL in the surge line. When the core coolant moves to the primary coolant system and remains there, the reactor core water level decreases. Thus, characteristics of CCFL in the surge line must be well understood for safety evaluation of the mid-loop operation.

Takeuchi et al. [4] calculated CCFL characteristics for a slightly inclined surge line of an AP600 using the momentum equations for steam and water. They reported that (1) CCFL in a vertical pipe is more dominant than that in a slightly inclined pipe, (2) the horizontal elbow increases 
the falling water volume, and (3) CCFL in the vertical pipe is the most dominant among various CCFLs taking place at different locations in the surge line. Although their prediction overestimated the falling water volume compared with the small break LOCA (Loss of Coolant Accident) data conducted at the AP600-scale test facility (APEX) $[5,6]$, there are no experimental data for validating their predictions. The surge line consists of a vertical pipe, a vertical elbow and an inclined pipe with several elbows. The flow in the surge line is very complicated due to its complex geometry, and therefore, it is difficult to apply the data and knowledge of CCFL obtained in a simple geometry such as straight pipes and ducts to CCFL in the surge line.

In this study, we carried out experiments using air and water in a 1/10-scale model and measured CCFL characteristics in the surge line. Effects of inclination angle and elbows in the surge line on CCFL characteristics were also investigated.

\section{Experimental Setup}

Figure 1 shows the experimental setup. It consists of the lower tank corresponding to a reactor vessel, the surge line, the upper tank simulating a pressurizer, and the air and water supply systems. The surge line is made of acrylic resin for the observation of flow pattern in the pipe. The internal diameter is $30 \mathrm{~mm}$. The geometry of the surge line is shown in Figure 2. Air is supplied through the sidewall of the lower tank. Water is supplied through the bottom face of the upper tank. They form a countercurrent flow in the surge line. The elbow is made of two acrylic blocks with semicircular grooves to keep the channel cross-section circle. At a constant flow rate $Q_{L \text { in }}$ of water supplied to the upper tank, the flow rate $Q_{L}$ of water falling into the lower tank was measured at each gas flow rate $Q_{G}$ to obtain a relationship between $Q_{L}$ and $Q_{G}$. The $Q_{L}$ was measured not only by increasing $Q_{G}$ but also by decreasing $Q_{G}$ to check a possibility of hysteresis in CCFL. The experimental ranges were $J_{L \text { in }}\left(=4 Q_{L \text { in }} / \pi D^{2}\right)=$ $0.02-0.12 \mathrm{~m} / \mathrm{s}$ and $J_{G}\left(=4 Q_{G} / \pi D^{2}\right)=0-5.5 \mathrm{~m} / \mathrm{s}$. CCFL data were plotted by using the dimensionless gas and liquid volumetric fluxes, $J_{G}^{*}$ and $J_{L}^{*}$, given by [6]

$$
J_{k}^{*}=J_{k}\left\{\frac{\rho_{k}}{g D\left(\rho_{L}-\rho_{G}\right)}\right\}^{1 / 2}, \quad(k=G, L),
$$

where $J$ is the superficial velocity, $\rho$ the density, $g$ the acceleration of gravity, and $D$ the pipe diameter. The subscripts $G$ and $L$ denote the gas and liquid phases, respectively. The inclination angle $\theta$ of the surge line was changed from 0.0 to $5.0 \mathrm{deg}$. $(0.0,0.6,1.0,2.0$ and $5.0 \mathrm{deg}$.) to investigate the effects of $\theta$ on CCFL characteristics. We also measured CCFL characteristics by replacing the surge line with the straight pipe shown in Figure 3 to examine effects of elbows.

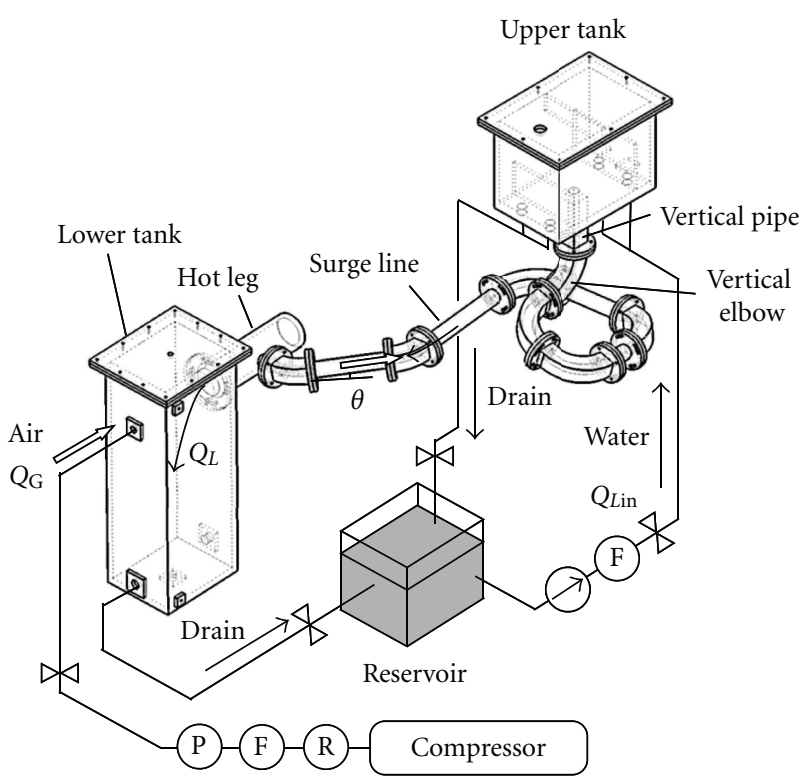

(F: flow meter, P: pressure gage, R: regulator)

Figure 1: Experimental setup (Surge line).

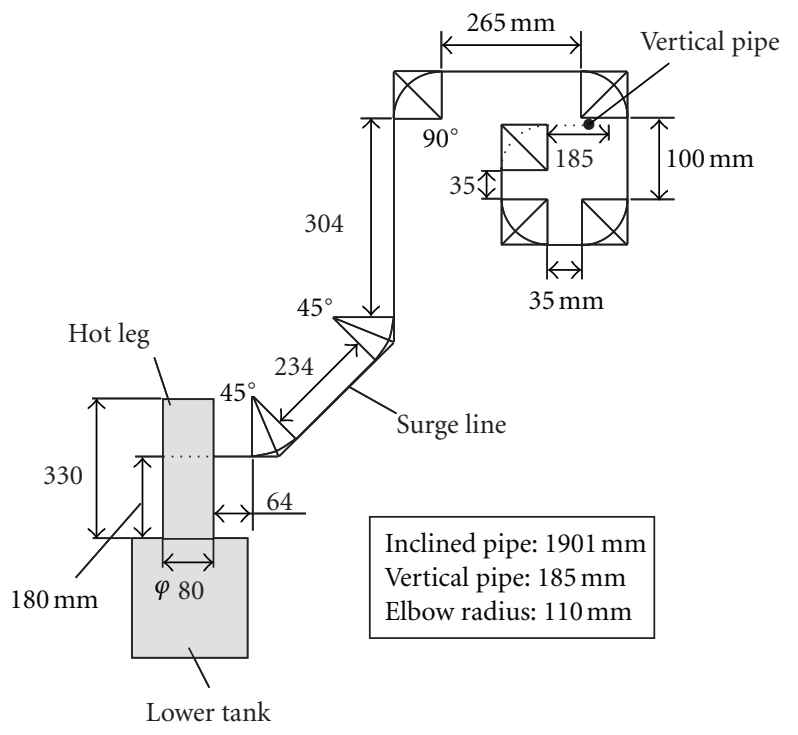

FIGURE 2: Geometry of surge line.

\section{Results and Discussion}

3.1. Classification of CCFL. Depending on the inclination angle $\theta$ and $J_{G}$, CCFL took place at three different locations, that is, at the upper junction, in the surge line, and at the lower junction as shown in Figure 4. Hereafter, CCFL at the upper junction between the surge line and the upper tank, in the surge line and that at the lower junction between the surge line and the hot leg will be referred to as CCFL-U, CCFL-S, and CCFL-L, respectively.

In CCFL-U, the flow limitation occurs only at the upper junction of the surge line as shown in Figure 5(a), and 


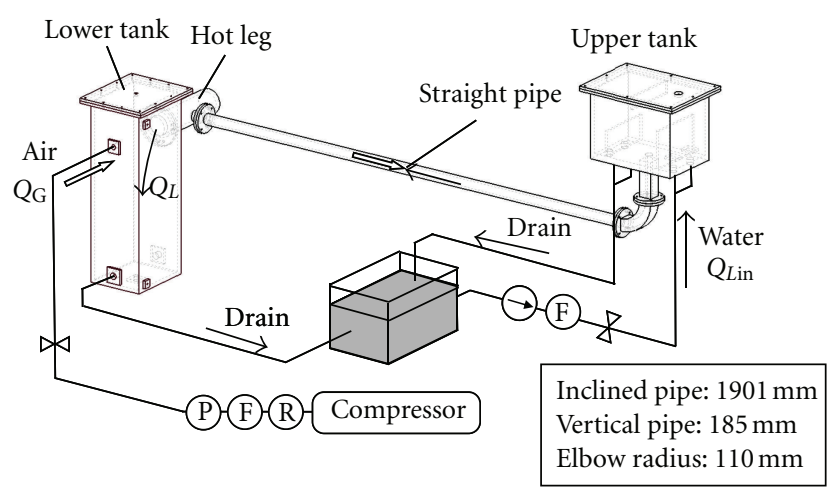

(F: flow meter, P: pressure gage, R: regulator)

FIgURE 3: Experimental apparatus with straight pipe.

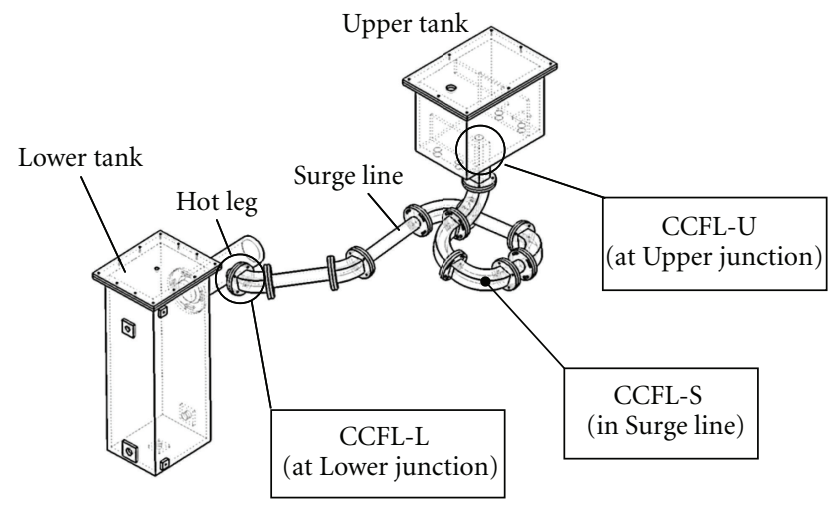

FIgURE 4: Flow limitation locations and CCFL classification.

therefore, water in the surge line and at the lower junction smoothly flows toward the lower tank. To the contrary, the flow limitation occurs not only at the upper junction but also in the surge line in CCFL-S as shown in Figure 5(b). Waves are generated in the surge line and move toward the upper junction. In CCFL-L, the flow limitation occurs at the lower junction as well as at the upper junction. Water is accumulated near the lower junction to form large liquid slugs and periodically flows back toward the upper junction as shown in Figure 5(c).

3.2. CCFL Characteristics at Reference Condition. Figure 6 shows CCFL characteristics at $J_{\text {Lin }}=0.07 \mathrm{~m} / \mathrm{s}$ and $\theta=$ $0.6 \mathrm{deg}$. As $J_{G}^{*}$ increases from zero, flow limitation takes place when $J_{G}^{*}$ reaches a certain critical value (point $\mathrm{A}$ in Figure 6). At point $A$, waves form in the surge line and move toward the upper junction. At the same time, $J_{L}^{*}$ suddenly decreases to the flooding point (point B in Figure 6), that is, CCFL-S occurs. Further increase in $J_{G}^{*}$ reduces $J_{L}^{*}$, and all the water returns to the upper tank at the flow reversal point (point $\mathrm{C}$ in Figure 6). When $J_{G}^{*}$ is decreased from the flow reversal point $C, J_{L}^{*}$ gradually increases as shown in Figure 6. The minimum $J_{G}^{*}$ observed in the decreasing process is smaller than $J_{G}^{*}$ at the flooding point $\mathrm{B}$. Thus, hysteresis exists in the CCFL characteristics. The hysteresis is caused by the difference in the presence of initial waves on the gas-liquid interface. There is, however, no difference in the dependence of $J_{L}^{*}$ on $J_{G}^{*}$ between the processes of increasing and decreasing $J_{G}^{*}$.

3.3. Effects of $\theta$. Figure 7 shows CCFL characteristics at various $\theta$. At $\theta=0.0$ and 0.6 deg., only CCFL-S takes place at any values of $J_{G}$. At $\theta=1.0$ deg., CCFL disappears in the surge line, and therefore, it is classified as CCFL- $U$ when $J_{G}$ is low, whereas CCFL-S occurs at high $J_{G}$. This disappearance of CCFL in the surge line is due to the enhancement of water drainage by increasing $\theta$. At $\theta=2.0$ and 5.0 deg., CCFL$\mathrm{L}$ appears instead of CCFL-S at high $J_{G}$. This indicates that the flow limitation at the lower junction becomes dominant because CCFL in the surge line is mitigated by the increase in $\theta$. At low $J_{G}$, CCFL-U occurs not only for $\theta=2.0$ and $5.0 \mathrm{deg}$. but also for $\theta=1.0 \mathrm{deg}$. These results show that type of CCFL depends on $\theta$ and $J_{G}^{*}$, and the dependence of the relation between $J_{G}^{*}$ and $J_{L}^{*}$ on $\theta$ is different among CCFL-S, CCFL-U, and CCFL-L.

Figure 8 shows characteristics of CCFL-S at various $\theta$. A small change in $\theta$ causes a large change in the falling water flow rate, that is, the dependence of CCFL-S on the inclination angle is very large. The increase in $\theta$ results in the mitigation of flow limitation in the surge line due to the enhancement of water drainage. Hence, CCFL-S occurs only at low $\theta$.

Figure 9 shows characteristics of CCFL-L at various $\theta$. CCFL-L is also affected by $\theta$, and the flow limitation becomes weaker as $\theta$ increases. The dependence of CCFL-L on the inclination angle, however, is weaker than that of CCFL-S. Since the holdup at the lower junction depends not only on the water velocity along the surge line but also on the velocity of water falling into the lower tank, the weak dependence of the falling water velocity on $\theta$ might be a cause of the small dependency of CCFL-L on $\theta$.

Figure 10 shows characteristics of CCFL-U at various $\theta$. CCFL- $U$ is the limitation at the upper junction between the upper tank and the vertical pipe, and the gravity force acting on the water along the surge line (the vertical pipe) is $\rho g$ cos $\theta$. Hence, CCFL-U has a very weak dependence on $\theta$ at small $\theta$ as shown in Figure 10.

These experimental results confirm that the effect of $\theta$ on CCFL depends on the flow direction, that is, the effect is large for the nearly horizontal flow in the surge line, small for the vertical flow at the upper junction, and intermediate for the flow at the lower junction at which the flow changes its direction from horizontal to vertical directions. The CCFL in the surge line is determined by the most strong flow limitation among CCFL-S, CCFL-U and CCFL-L.

3.4. Onset of Flooding. The gas volumetric flux at the onset of flooding is important information when designing surge lines. Figure 11 shows the onset of flooding measured by increasing the gas volumetric flux. Flooding-S, Flooding-U, and Flooding-L in Figure 11 represent that the flooding takes place in the surge line, at the upper junction and at the lower 

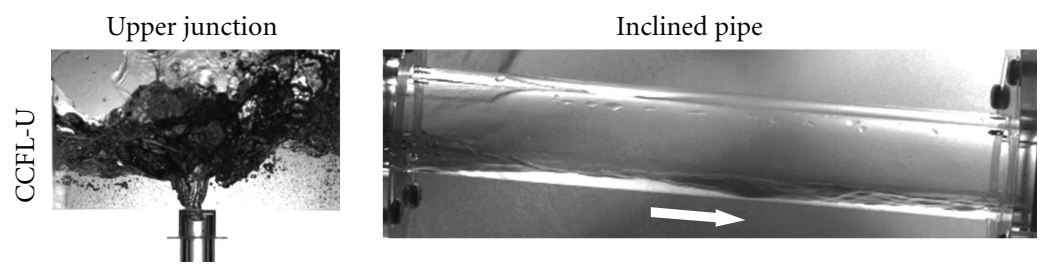

(a) $\theta=5.0 \mathrm{deg}, J_{\operatorname{Lin}}=0.07 \mathrm{~m} / \mathrm{s}, J_{G}=3.2 \mathrm{~m} / \mathrm{s}$

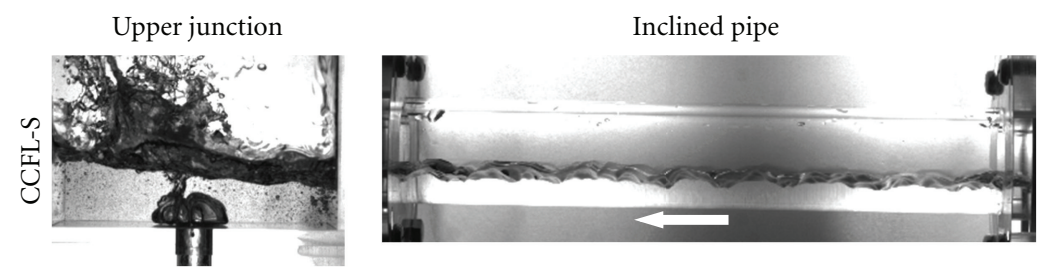

(b) $\theta=0.6 \mathrm{deg}, J_{L \text { in }}=0.07 \mathrm{~m} / \mathrm{s}, J_{G}=3.8 \mathrm{~m} / \mathrm{s}$
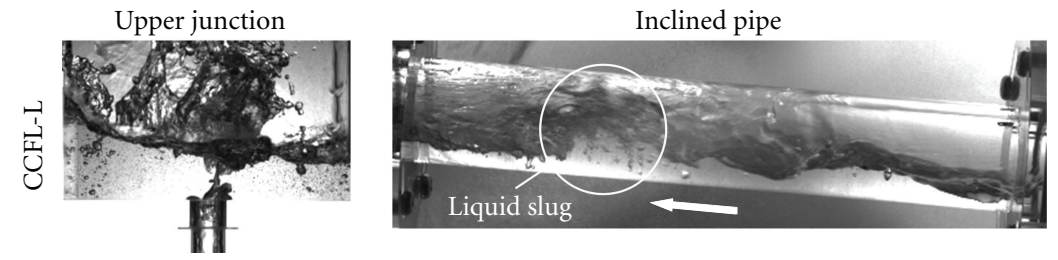

(c) $\theta=5.0 \mathrm{deg}, J_{\text {Lin }}=0.07 \mathrm{~m} / \mathrm{s}, J_{G}=5.5 \mathrm{~m} / \mathrm{s}$

Figure 5: Typical flow pattern at CCFL conditions.

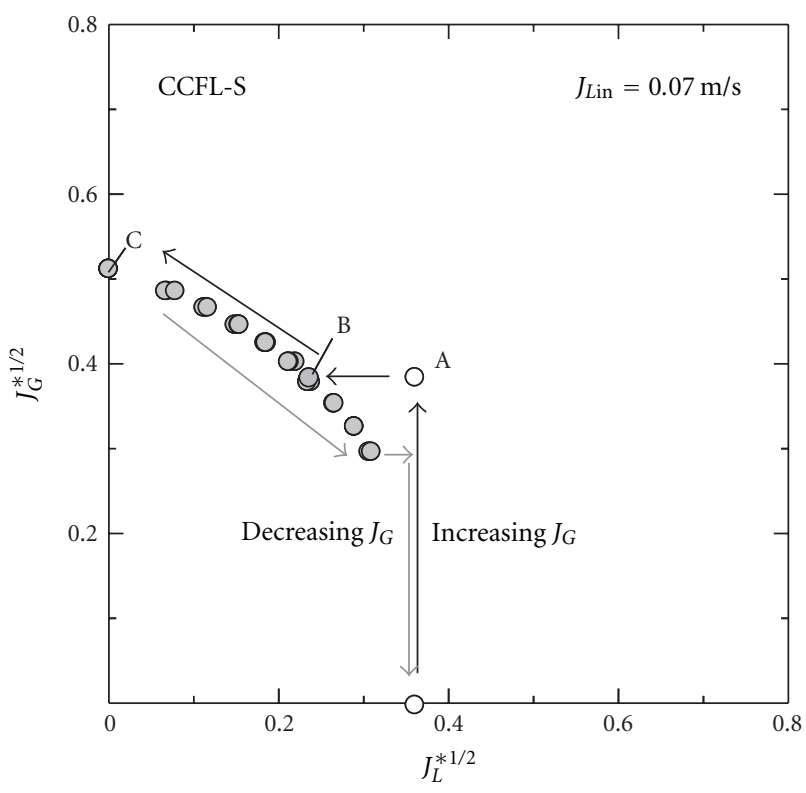

Figure 6: CCFL characteristics $(\theta=0.6$ deg.).

junction, respectively. The line in Figure 11 is drawn by using the Wallis's model [7-9]:

$$
\sqrt{J_{G}^{*}}+\sqrt{J_{L i n}^{*}}=1
$$

The $J_{G}^{*}$ at the onset of flooding decreases as $J_{\text {Lin }}^{*}$ increases, and $J_{G}^{*}$ increases with $\theta$. The flooding always occurs in the surge line at low $\theta$. On the other hand, at high $\theta$, it occurs at the

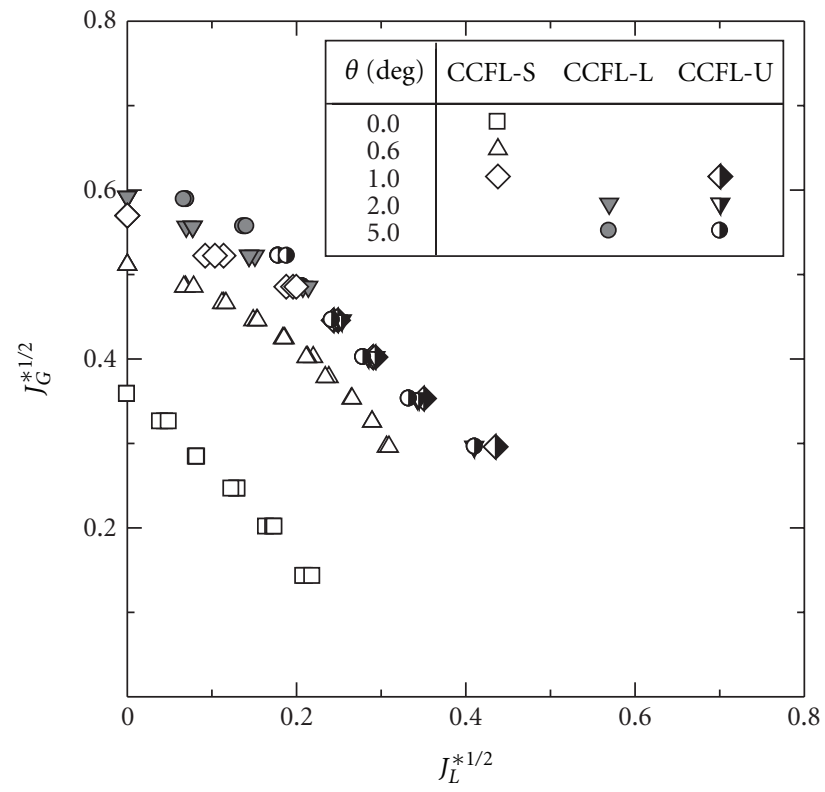

Figure 7: CCFL characteristics (effects of $\theta$ ).

lower junction when $J_{\text {Lin }}^{*}$ is low and at the upper junction at high $J_{\text {in }}^{*}$. Since the flooding at the upper junction is similar to that in a vertical pipe, the points of Flooding- $\mathrm{U}$ are not far from (2).

3.5. Effects of $J_{\text {Lin }}$. Figure 12 shows the CCFL characteristics under three different $J_{L \text { in }}$ conditions $\left(J_{\text {Lin }}=0.02,0.07\right.$, and 


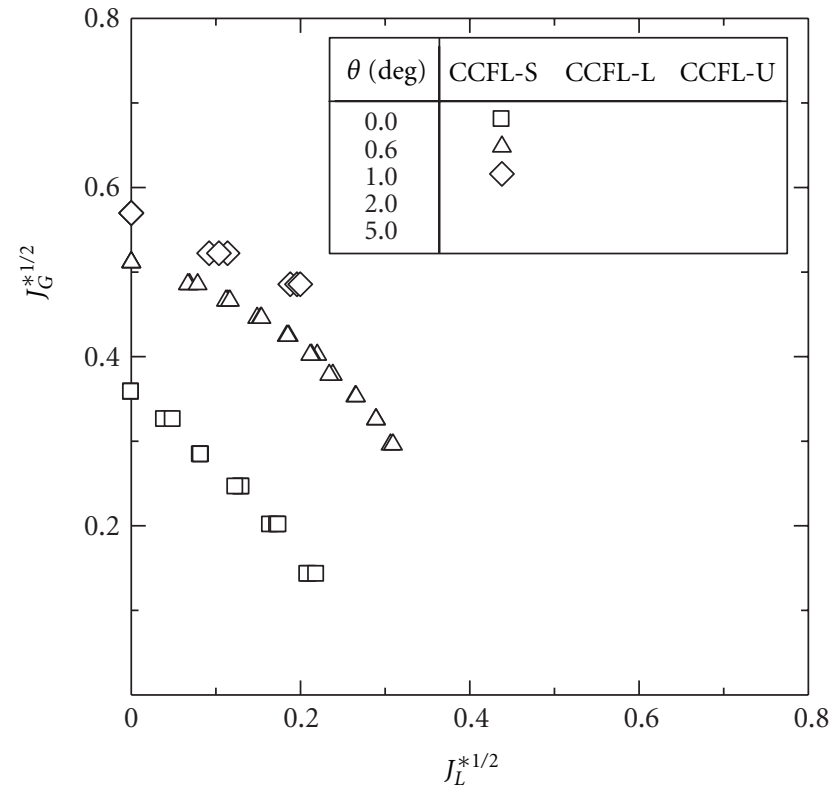

Figure 8: CCFL-S characteristics (effects of $\theta$ ).

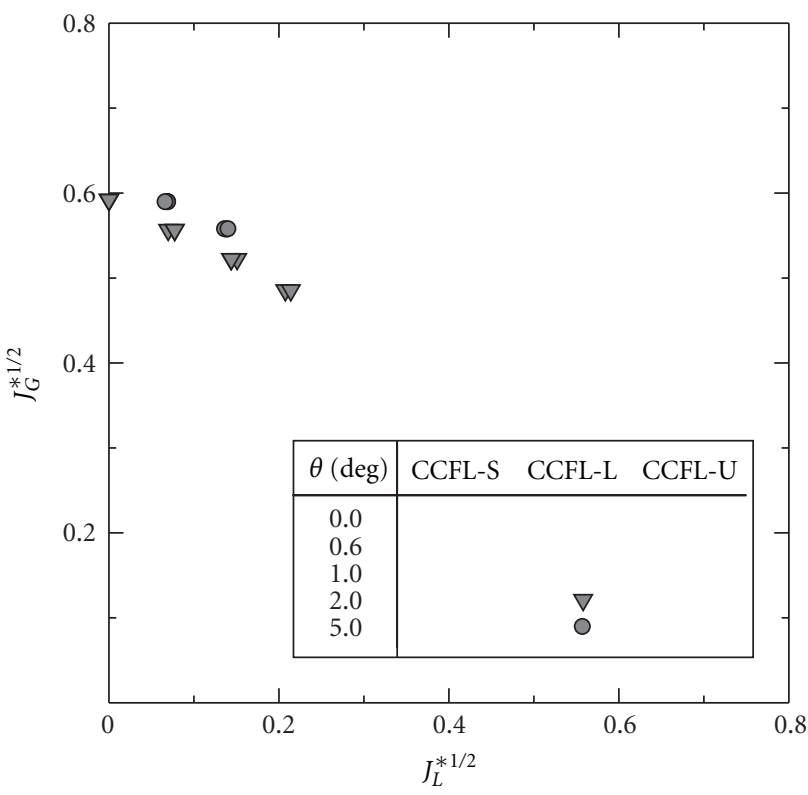

Figure 9: CCFL-L characteristics (effects of $\theta$ ).

$0.12 \mathrm{~m} / \mathrm{s}$ ) for $\theta=0.6$ and $5.0 \mathrm{deg}$. The volumetric flux $J_{L}^{*}$ of the falling water does not depend on the volumetric flux $J_{L \text { in }}$ of the supplied water in the upper tank, irrespective of $\theta$ and a type of CCFL (CCFL-S, CCFL-U, and CCFL-L). This is because the water level in the upper tank depends not on $J_{L \text { in }}$ but on the height of the partition in the upper tank when the flow limitation takes place.

3.6. Effects of Elbows. Figure 13 shows comparisons of CCFL characteristics between the surge line and the straight pipe. CCFL in the straight pipe is also classified into CCFL-S,

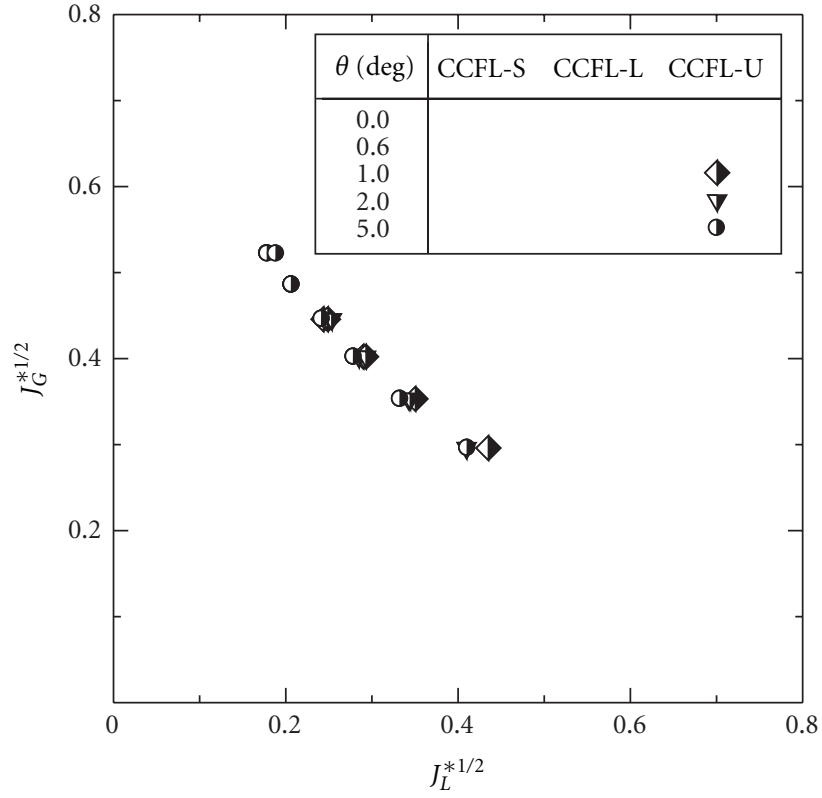

FIgURE 10: CCFL-U characteristics (effects of $\theta$ ).

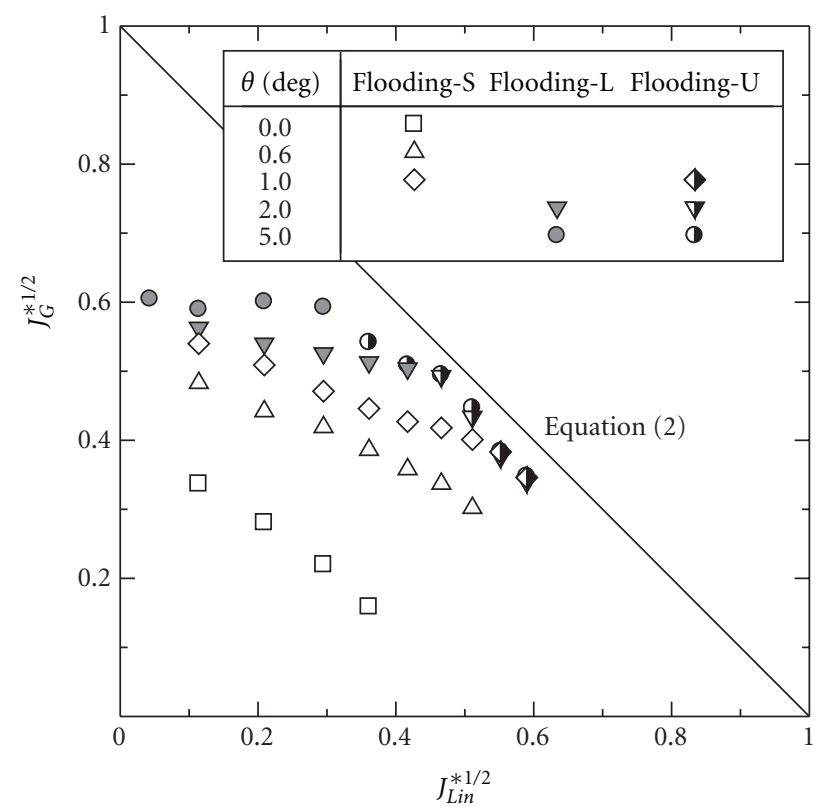

Figure 11: Onset of flooding.

CCFL-U, and CCFL-S. CCFL-S occurs at low $\theta$ whereas CCFL-U, and CCFL-L appear at high $\theta$. The flow limitation in the surge line is stronger than that in the straight pipe as shown in Figure 13(a). The elbows, therefore, enhance the flow limitation in the surge line, which contradicts the predictions obtained by Takeuchi et al. [4]. They explained that centrifugal force in the elbow section stabilizes the gasliquid interface and inhibits the flow limitation. However, the centrifugal force would make the liquid film thinner and increase the wall friction. In addition, the presence of elbows would increase pressure drop in the line, in other words, 


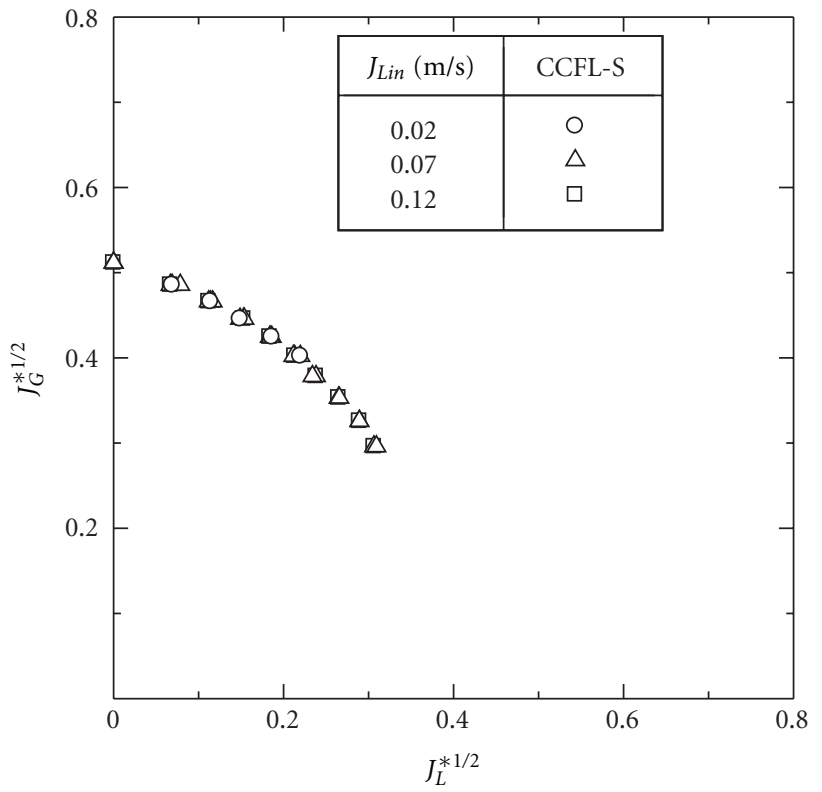

(a) $\theta=0.6 \mathrm{deg}$

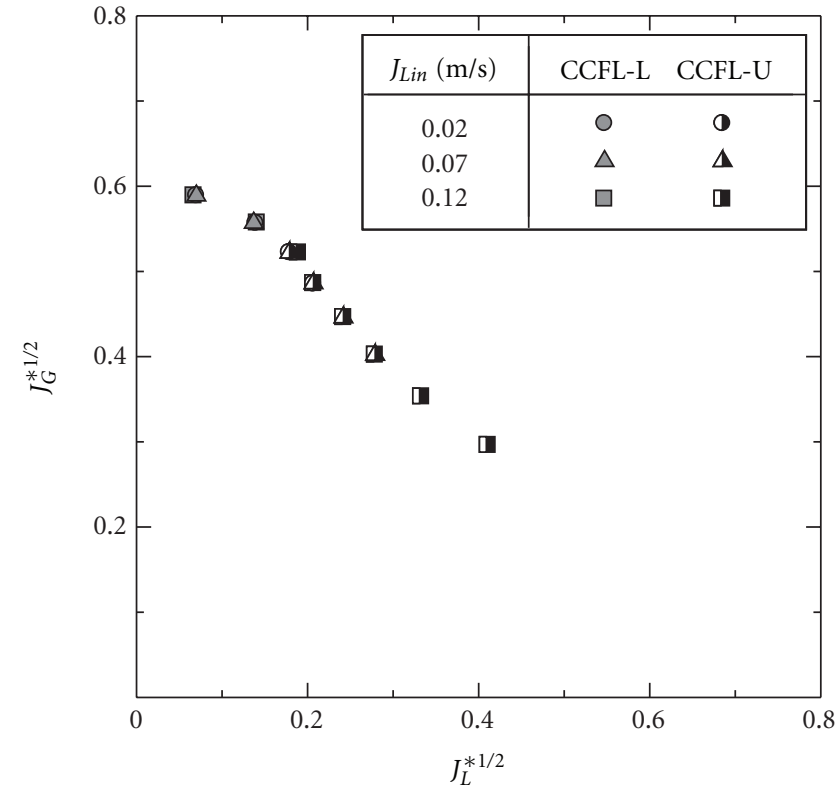

(b) $\theta=5.0 \mathrm{deg}$

FIgURE 12: CCFL characteristics (effects of $J_{\text {Lin }}$ ).

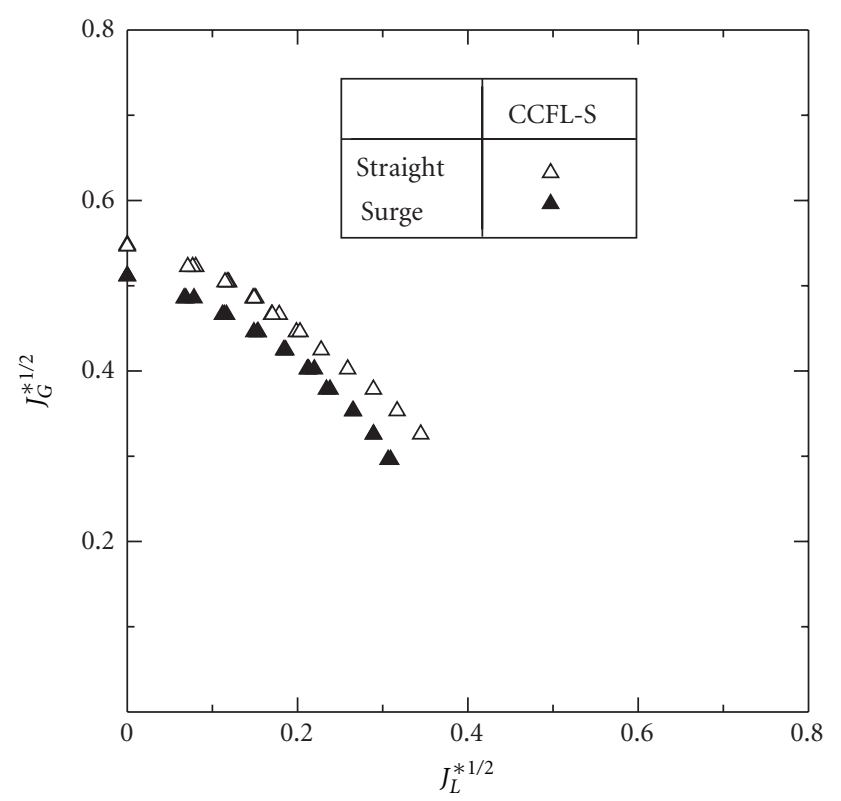

(a) $\theta=0.6 \mathrm{deg}$

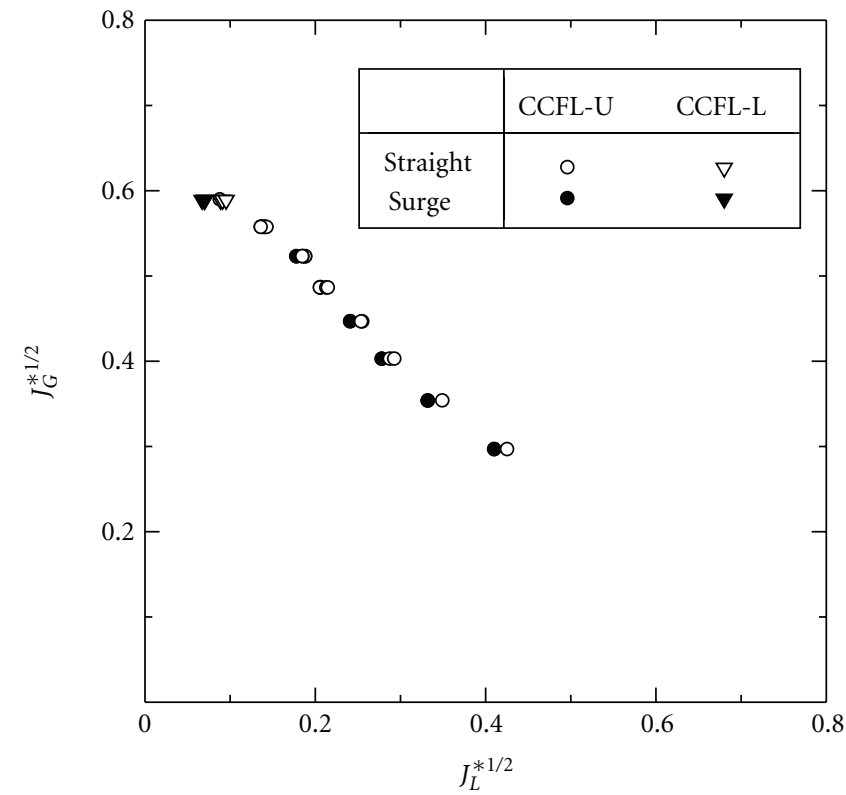

(b) $\theta=5.0 \mathrm{deg}$

FIgURE 13: Effect of elbows on CCFL characteristics.

increases the force acting on water in the upstream direction. These effects would result in enhancement of flow limitation. The present result, therefore, supports the latter speculation rather than Takeuchi's one. On the other hand, CCFL-U and CCFL-L do not depend on the presence of elbows as shown in Figure 13(b). This is because the flow limitation occurs at the junctions, and therefore, it has no relation with the elbows in the surge line.
3.7. Discussion on Effects of Size and Fluid Properties on CCFL Characteristics. Minami et al. [10] measured CCFL characteristics in a scale-down model of PWR hot leg and confirmed thorough comparisons with literature [11-14] that the effects of the size and fluid properties are small. Since CCFL-L and CCFL-S in the surge line are similar to CCFL in the hot leg, this result implies that their dependence on the size and fluid properties is also small. On the other 
hand, CCFL- $\mathrm{U}$ is similar to CCFL in a vertical pipe. Many researches, which are summarized in textbooks $[7,15,16]$, have been carried out for CCFL in a vertical pipe. These researches indicate that the Kutateladze number is more appropriate than the dimensionless volumetric flux $J_{k}^{*}$ for large diameter tubes, and that the fluid properties can be taken into account by using the Bond number, viscosity ratio and/or Grashof number. This kind of knowledge can be utilized when applying the present results to a system with different pipe sizes or different fluid properties.

\section{Conclusions}

Countercurrent air-water flow in a scale-down model of a PWR pressurizer surge line was measured to understand characteristics of countercurrent flow limitation, CCFL. As a result, the following conclusions were obtained.

(1) CCFL takes place at three different locations, that is, at the upper junction, in the surge line, and at the lower junction. CCFL characteristics are governed by the most dominating flow limitation among the three.

(2) CCFL characteristics depend on the inclination angle of the surge line and the air flow rate. The effects of inclination angle on CCFL depend on the flow direction, that is, the effect is large for the nearly horizontal flow in the surge line, small for the vertical flow at the upper junction, and intermediate for the flow at the lower junction at which the flow changes its direction from horizontal to vertical directions.

(3) The presence of elbows enhances the flow limitation in the surge line, whereas the flow limitations at the upper and lower junctions do not depend on the presence of elbows.

\section{Nomenclature}

$D$ : Pipe diameter $[\mathrm{m}]$

$g$ : Acceleration of gravity $\left[\mathrm{m} / \mathrm{s}^{2}\right]$

$J$ : Volumetric flux $[\mathrm{m} / \mathrm{s}]$

$J^{*}$ : Dimensionless volumetric flux

Q: Volume flow rate $\left[\mathrm{m}^{3} / \mathrm{s}\right]$

$\rho:$ Density $\left[\mathrm{kg} / \mathrm{m}^{3}\right]$

$\theta$ : Angle of inclination [deg.].

\section{Subscripts}

G: Gas phase

$L$ : $\quad$ Liquid phase

Lin: Liquid phase supplied to the upper tank.

\section{References}

[1] N. Minami, D. Kataoka, A. Tomiyama, S. Hosokawa, and M. Murase, "Countercurrent gas-liquid flow in a rectangular channel simulating a PWR hot leg (1) flow pattern and CCFL characteristics," Japanese Journal of Multiphase Flow, vol. 22, no. 4, pp. 403-412, 2008 (Japanese).

[2] N. Minami, M. Murase, D. Nishiwaki, and A. Tomiyama, "Countercurrent gas-liquid flow in a rectangular channel simulating a PWR hot leg (2) analytical evaluation of countercurrent flow limitation," Japanese Journal of Multiphase Flow, vol. 22, no. 4, pp. 413-422, 2008 (Japanese).

[3] H. Nakamura, J. Katayama, and Y. Kukita, "Loss of residual heat removal (RHR) event during PWR mid-loop operation: ROSA-IV/LSTF experiment without opening on primary loop pressure boundary," American Society of Mechanical Engineers, Fluids Engineering Division (Publication) FED, vol. 140, pp. 916, 1992.

[4] K. Takeuchi, M. Y. Young, and A. F. Gagnon, "Flooding in the pressurizer surge line of AP600 plant and analyses of APEX data," Nuclear Engineering and Design, vol. 192, no. 1, pp. 45$58,1999$.

[5] L. E. Hochreiter, S. V. Fanto, L. E. Conway, and L. K. Lau, "Integral testing of the AP600 passive emergency core cooling systems," Journal of Power and Energy, vol. 207, no. 4, pp. 259268, 1993.

[6] J. N. Reyes, "Scaling Analysis for the OSU AP600 Integral Systems and Long Term Cooling Facility," OSU-NE-9204, 1992.

[7] G. B. Wallis, One Dimensional Two-Phase Flow, McGraw Hill, New York, NY, USA, 1969.

[8] S. Levy, Two-Phase Flow in Complex Systems, Wiley Interscience, 1999.

[9] G. F. Hewitt and G. B. Wallis, ASME Multi-Phase Flow Symposium, ASME, Philadelphia, Pa, USA, 1963.

[10] N. Minami, D. Nishiwaki, T. Nariai, A. Tomiyama, and M. Murase, "Countercurrent gas-liquid flow in a PWR hot leg under reflux cooling (I) air-water tests for 1/15-scale model of a PWR hot leg," Journal of Nuclear Science and Technology, vol. 47, no. 2, pp. 142-148, 2010.

[11] H. J. Richter, G. B. Wallis, K. H. Carter et al., "Deentrainment and Countercurrent Air-Water Flow in a Model PWR HotLeg," NRC-0193-9, U.S. Nuclear Regulatory Commission, 1978.

[12] A. Ohnuki, "Experimental study of counter-current two-phase flow in horizontal tube connected to an inclined riser," Journal of Nuclear Science and Technology, vol. 23, no. 3, pp. 219-232, 1986.

[13] A. Ohnuki, H. Adachi, and Y. Murao, "Scale effects on countercurrent gas-liquid flow in a horizontal tube connected to an inclined riser," Nuclear Engineering and Design, vol. 107, no. 3, pp. 283-294, 1988.

[14] F. Mayinger, P. Weiss, and K. Wolfert, "Two-phase flow phenomena in full-scale reactor geometry," Nuclear Engineering and Design, vol. 145, no. 1-2, pp. 47-61, 1993.

[15] P. B. Whalley, Boiling Condensation and Gas-Liquid Flow, Oxford University Press, New York, NY, USA, 1987.

[16] J. M. Delhaye, M. Giot, and M. L. Riethmuller, Thermohydraulics of Two-Phase Systems for Industrial Design and Nuclear Engineering, Hemisphere, New York, NY, USA, 1981. 

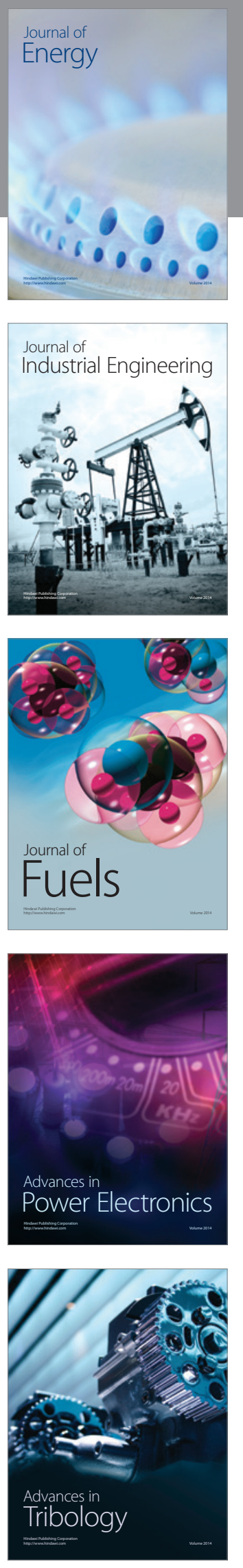
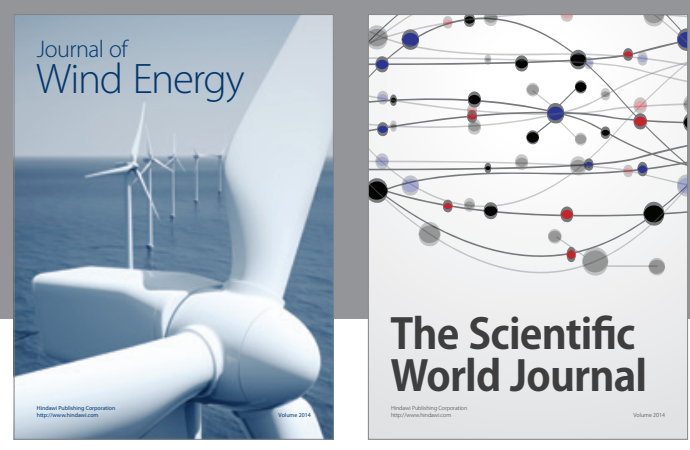

The Scientific World Journal

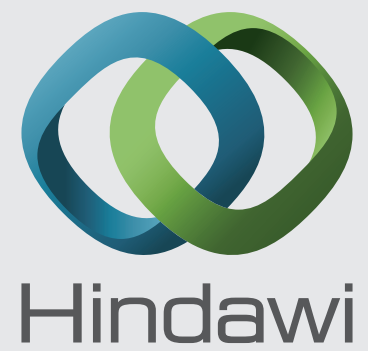

Submit your manuscripts at http://www.hindawi.com
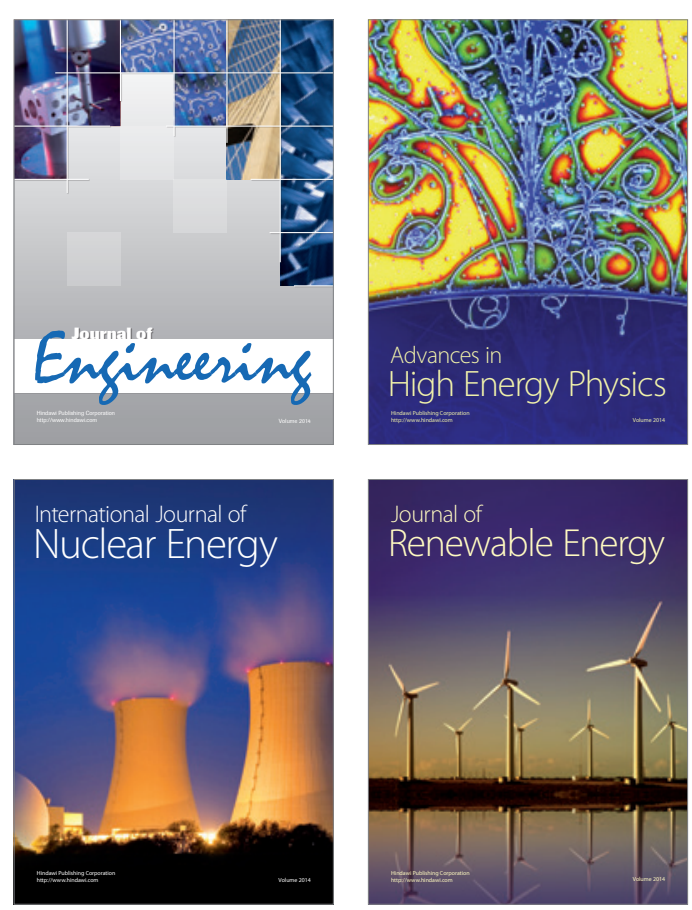

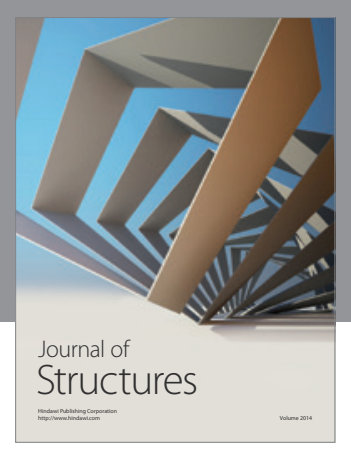

Rotating
Mechinery
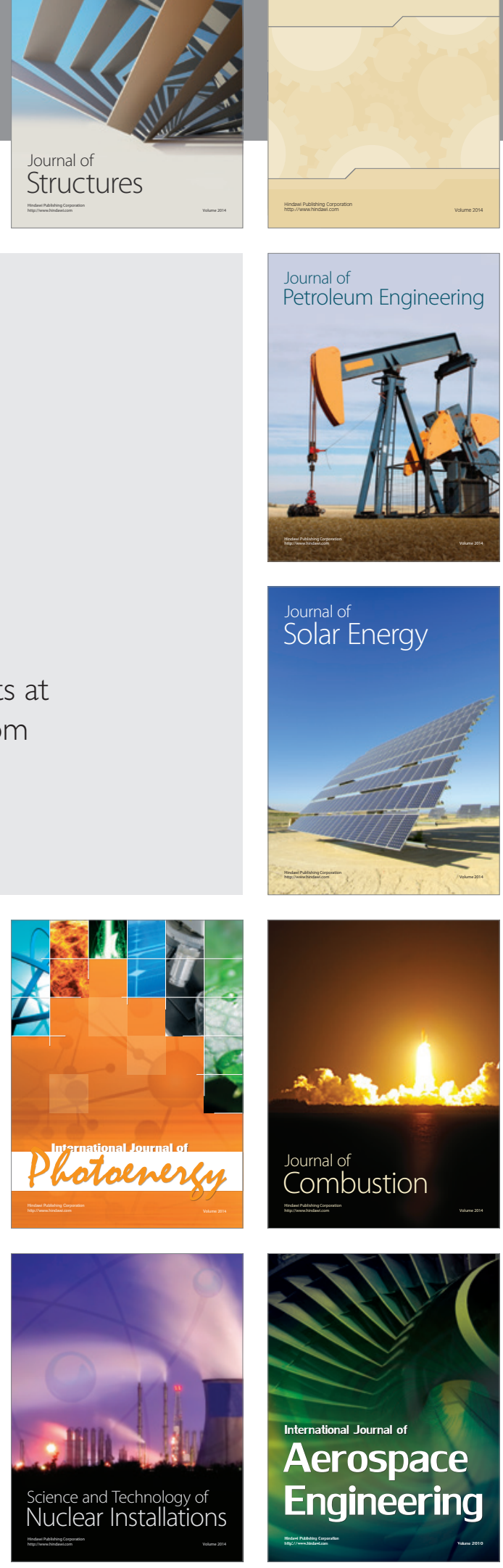